\title{
Length-Based Connectivity Metrics and Their Ecological Interpretation*
}

\author{
C.M. Baker \\ School of BioSciences, The University of Melbourne, Victoria 3010, Australia \\ cbaker2@student.unimelb.edu.au \\ B.D. Hughes and K.A. Landman \\ School of Mathematics and Statistics, The University of Melbourne, Victoria 3010, Australia \\ barrydh@unimelb.edu.au, kerryl@unimelb.edu.au
}

\begin{abstract}
Metrics that quantify habitat connectivity and fragmentation in landscape ecology are examined, as well as the relations between them and their interpretation. The radius of gyration, defined here as the root-mean-square distance of habitat patches from the center of the habitat, has special significance from an ecological point of view that other metrics lack. This metric has been incorrectly used in the field of landscape ecology - its definition is different from its definition in every other field, causing it to lose its ecological meaning. Inconsistencies in terminology between landscape ecology and other fields raise dangers of misinterpretation of metrics.
\end{abstract}

Subject headings: fragmented habitats, landscape metrics, radius of gyration, GYRATE, FRAGSTATS

\section{Introduction}

Habitat change is a key driver of biodiversity loss (Duraiappah et al. 2005) and quantifying how land use change influences habitat connectivity and fragmentation is a challenging problem in landscape ecology. A huge number of landscape metrics exist (Wu 2013). Many of these are convenient to measure and are capable of identifying patterns in landscapes, but are unable to relate these patterns to underlying processes (Ewers \& Didham 2007; Kupfer 2012; Moilanen \& Hanski 2001). The relationship between particular metrics and changes in a landscape has been previously addressed (Hargis et al. 1998), but a clear ecological interpretation is not available for every common metric, while the use of metrics without a clear ecological relevance can lead to meaningless results ( $\mathrm{Li} \& \mathrm{Wu}$ 2004; White et al. 2014).

${ }^{*}$ This is an author-prepared preprint equivalent in content to the version published in Ecological Indicators $\mathbf{5 8}$ (2015) 192-198
The availability of software to facilitate computing landscape metrics, such as the widely used FRAGSTATS package (McGarigal et al. 2012) has greatly empowered ecologists and geographers, but raised the possibility of inappropriate use of quantitative data, and especially of misinterpretation of the ecological significance of measurements.

One metric in particular has been misused in the ecological literature, the 'radius of gyration'. Keitt et al. (1997) and Riitters et al. (1995) were early users in ecology of what they describe as the radius of gyration, but the equations given differ from one another and neither coincides with the definition of radius of gyration that is in use everywhere except in landscape ecology. The radius of gyration was originally a physics term, used as a measure of the distribution of mass of an object (Synge \& Griffith 1959). Keitt et al. (1997) give two ecological interpretations of the metric, but neither of them corresponds to any definition of the radius of gyration. However, if the radius of gyration is calculated correctly, it can give a 

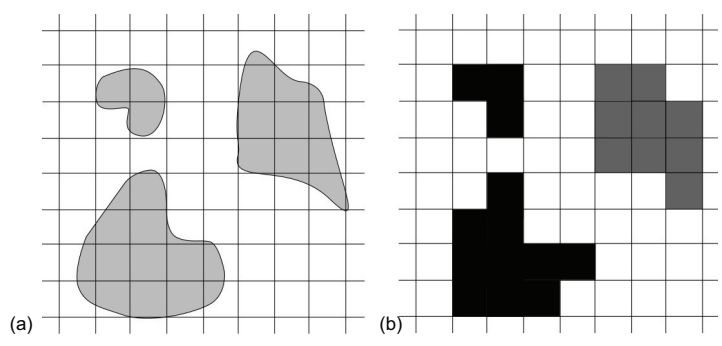

Fig. 1.- Habitat regions and their discrete representation. (a) The smooth grey regions represent suitable habitat for a species. (b) The centers of the shaded squares define the discrete habitat points, which may be characterized quantitatively en masse, or by first computing metrics for the clusters separately, and then constructing a cluster-size weighted average. Depending on the ability of the species under study to migrate across inhospitable territory, the three clusters could be appropriately analysed as a single habitat, or as two habitats (both black clusters as one habitat, and the dark grey cluster as a second, distinct habitat), or as three distinct habitats.

meaningful measurement of an animal's dispersal within a habitat patch.

In this note, we discuss six landscape metrics with the units of length, as length metrics are suitable for calculating dispersal range (which is by definition a length). One of these is included in FRAGSTATS as the statistic GYRATE, but all are readily computable from categorical maps. We address the relations between these six distance measures and their ecological significance, and in particular the difference between GYRATE and "radius of gyration" that arises in other disciplines. We also consider a potentially ecologically relevant landscape metric that standard software is unlikely to deliver, based on the time taken for a randomly exploring animal to reach the edge of its allowed habitat, which produces a distance metric quite distinct from the others discussed here.

\section{Six distance scales for discrete data}

In this section we consider six landscape metrics that can be easily calculated. We show how they are derived and why they are potentially useful. Finally, we show that only two of these metrics are related, producing an ecological interpretation for the radius of gyration. Meaningful landscape metrics (e.g. to characterise habitat configuration) should be free from any dependence on choices of origin of coordinates or idiosyncrasies in definitions. It is natural, therefore, to compute metrics that arise from some form of average of distances of arbitrary points from the center of the habitat, from the nearest boundary of the habitat, or in relation to the rest of the habitat. We discuss all of these below. The Appendix contains a discussion of these matters for continuous regions, whereas in this section we address discrete landscape data. For example, discrete data arises by covering the landscape with a regular grid (say a square mesh) and identifying the central point of a mesh cell that is filled with habitat (either completely, or to a specified fraction) as a discrete habitat point, which we refer to as a patch. This process of altering continuous regions to discrete landscape data is illustrated in Fig. 1.

If our discrete habitat consists of $N$ points $\left(\mathbf{r}_{i}\right.$, where $1 \leqslant i \leqslant N$ ) then the centroid or center of mass of the habitat is given by

$$
\mathbf{r}_{\mathrm{cm}}=\left(x_{\mathrm{cm}}, y_{\mathrm{cm}}\right)=\frac{1}{N} \sum_{i=1}^{N} \mathbf{r}_{i} .
$$

By analogy with conventional use in other disciplines, we define the radius of gyration $R_{\mathrm{g}}$ by

$$
R_{\mathrm{g}}^{2}=\frac{1}{N} \sum_{i=1}^{N}\left|\mathbf{r}_{i}-\mathbf{r}_{\mathrm{cm}}\right|^{2} .
$$

The term radius of gyration has its roots in physics, where both the discrete system equations (1) and (2) and their continuum analogues (discussed in the Appendix) arise. For a rigid body, the ratio of the moment of inertia about an axis to the total mass of that body is the square of the radius of gyration about the axis (Synge \& Griffith 1959). The moment of inertia determines the torque required to rotate the body with a specific angular acceleration. Eq. (2) corresponds to the definition of the radius of gyration for a rigid assembly of point masses (each with the same mass). This definition also agrees with that used in statistical physics in the context of the shape of random walks Hughes (1995) and percolation clusters (Hughes 1996; Stauffer \& Aharony 1994).

The radius of gyration can be calculated for objects in an arbitrary number of dimensions, but 
in the context of landscape metrics, we are interested in the two-dimensional case. For this special case, the radius of gyration is a natural length that characterizes the extent of a region: $R_{\mathrm{g}}$ is the root-mean-square (RMS) distance between habitat patches and the habitat centroid. Although the radius of gyration can be used directly whether or not the habitat is connected, if the total habitat consists of disconnected clusters, a finite number of isolated habitat clusters may be identified. If a habitat cluster contains $N(k)$ patches, where $k$ is the cluster's label, and has radius of gyration $R_{\mathrm{g}}(k)$, then an appropriate area-weighted average of the radii of gyration of the distinct habitats, perhaps naturally called the correlation length by analogy with statistical physics, is the length-scale $\xi$ defined by

$$
\xi^{2}=\frac{\sum_{k} R_{\mathrm{g}}(k)^{2} N(k)}{\sum_{k} N(k)} .
$$

If precedents in other disciplines are ignored, a plausible different measure of the typical distance of habitat points from the centroid is

$$
R_{*}=\frac{1}{N} \sum_{i=1}^{N}\left|\mathbf{r}_{i}-\mathbf{r}_{\mathrm{cm}}\right| .
$$

Here, and in subsequent discussion, a subscript asterisk on a parameter indicates that it is computed as a direct average, rather than as an RMS average. The FRAGSTATS package statistic GYRATE corresponds to $R_{*}$. For fragmented habitats, the analogue of Eq. (3) is

$$
\xi_{*}=\frac{\sum_{k} R_{*}(k) N(k)}{\sum_{k} N(k)} .
$$

In FRAGSTATS notation, $\xi_{*}=$ GYRATE_AM.

For any random variable $X$ with finite variance, the inequality $\left\langle(X-\langle X\rangle)^{2}\right\rangle \geqslant 0$ (where angle brackets denote expectation) ensures that $\left\langle X^{2}\right\rangle \geqslant\langle X\rangle^{2}$, with equality only possible in the trivial case where $X$ takes a unique value with probability 1 . Hence (excluding the trivial case of a habitat with only one patch), we have the strict inequality $R_{\mathrm{g}}>R_{*}$. The discrepancy between $R_{\mathrm{g}}$ and $R_{*}$ is illustrated in Fig. 2: it is quite sensitive to how the patches are arranged.

Another way to characterize habitat connectivity is to average in some way the separation of randomly chosen patches. To this end one may introduce the root-mean-square patch separation $\rho$ and the mean patch separation $\rho_{*}$ defined by

$$
\begin{aligned}
& \rho^{2}=\frac{1}{N^{2}} \sum_{i=1}^{N} \sum_{j=1}^{N}\left|\mathbf{r}_{i}-\mathbf{r}_{j}\right|^{2} ; \\
& \rho_{*}=\frac{1}{N^{2}} \sum_{i=1}^{N} \sum_{j=1}^{N}\left|\mathbf{r}_{i}-\mathbf{r}_{j}\right| .
\end{aligned}
$$

In addition to noting the strict inequality $\rho>\rho_{*}$ for discrete non-trivial habitats, we draw to the reader's attention the easily proved elegant result (Brissonette 1997; Creswick et al. 1992; Feder 1988; Hughes 1996):

$$
\rho^{2}=2 R_{\mathrm{g}}^{2}
$$

The radius of gyration tells us something directly about the separation of patches. This universal relation between RMS metrics is not mirrored for the corresponding direct average metrics: there is no shape-independent relation between $R_{*}$ and $\rho_{*}$.

Two other metrics that are easily computed for discrete habitats (though not tidily represented by formulae) are the RMS average and direct average distances of patches from the closest boundary: these distances could be computed either as straight-line distances, or as accumulated distances stepping between lattice patches.

In Table 1 we record the values of $R_{\mathrm{g}}$ (RMS distance from the centroid), $R_{*}, \rho$ (RMS distance between two points), $\rho_{*}$ and the RMS average and direct average straight-line distances from the closest boundary point computed for two continuous (rather than discrete) habitats, viz. a circle and a square. Except for the universal result that $R_{\mathrm{g}}=\sqrt{2} \rho$, there is no simple relationship between any pair of metrics.

\section{Discussion}

While many landscape metrics can be computed alongside the six we have considered in Section 2, it is important to ask which are likely to have greatest ecological relevance. There are reasons in the context of organism dispersion for favouring the RMS average $R_{\mathrm{g}}$ over the alternative metric $R_{*}$, as we now explain.

We consider the case of organisms which move at random and independently within the habitat, 

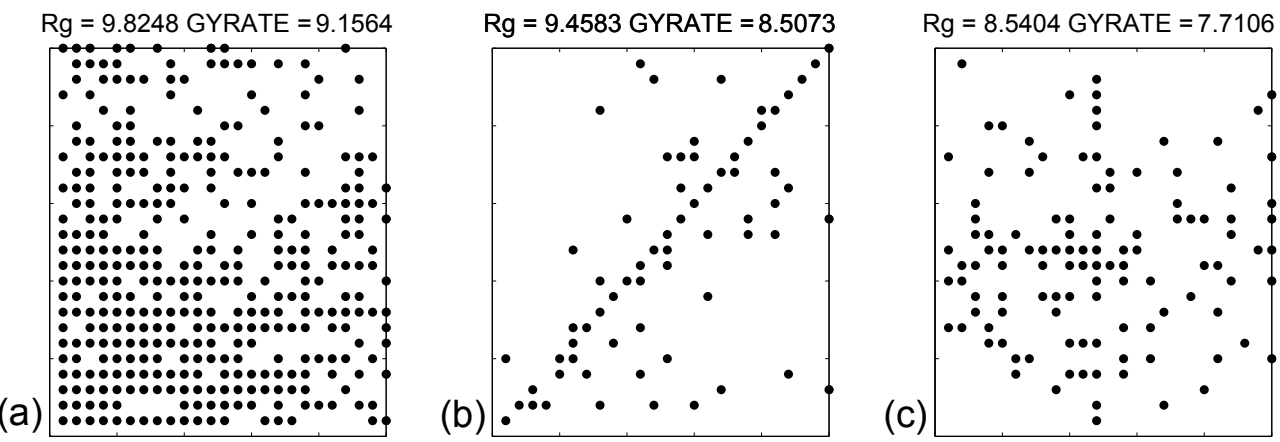

Fig. 2.- The radius of gyration $R_{\mathrm{g}}$ and $R_{*}=$ GYRATE for three different discretized habitats on $25 \times 25$ cell square grids. (a) The habitat is fairly dense and its patches are more likely to occur in the bottom left corner. (b) This habitat is sparser and patches are most likely to be on or near the diagonal. (c) This habitat is also fairly sparse, but patches are more likely to occur towards the center than the edges.

TABLE 1

Six distance SCAles Associated With TWO Simple Shapes. Values preceded by "=" are exact, while VALUES PRECEDED BY $\approx$ ARE CORRECT TO TWO DECIMAL POINTS (EXACT VALUES FOR SOME OF THESE INVOLVING INTEGERS AND surds are available, while others are obtained by numerical integration). Details are given in the Appendix.

\begin{tabular}{llll}
\hline \hline Shape of region & $\begin{array}{c}\text { Distance from the } \\
\text { centroid }\end{array}$ & $\begin{array}{l}\text { Distance from the } \\
\text { nearest boundary }\end{array}$ & $\begin{array}{c}\text { Distance between } \\
\text { two points }\end{array}$ \\
\hline circle of radius $a$ & $\begin{array}{l}\text { Mean } \approx 0.67 a \\
\text { RMS } \approx 0.71 a\end{array}$ & $\begin{array}{l}\text { Mean } \approx 0.33 a \\
\text { RMS } \approx 0.41 a\end{array}$ & $\begin{array}{l}\text { Mean } \approx 0.95 a \\
\text { RMS }=a\end{array}$ \\
\hline \multirow{2}{*}{ square of side $L$} & Mean $\approx 0.38 L$ & Mean $\approx 0.17 L$ & Mean $\approx 0.52 L$ \\
& RMS $\approx 0.41 L$ & RMS $=0.20 L$ & RMS $\approx 0.57 L$ \\
\hline
\end{tabular}

but do not cross its boundaries. The dispersal distance for an organism that starts at patch $\mathbf{r}$ and is found at patch $\mathbf{r}^{\prime}$ is simply $\left|\mathbf{r}-\mathbf{r}^{\prime}\right|$. In the discrete model, the organism dispersal can be modelled as a simple random walk on a lattice with steps that attempt to move out of the habitat aborted; in a continuum model the organism undergoes Brownian motion and the probability density function for its random location is computed from the diffusion equation with reflecting boundaries. In both cases, the probability distribution for an individual's location is uniform across the habitat in the long-term limit. Hence if the starting location of the organism is also random, the metrics $\rho$ and $\rho_{*}$ correspond to root-mean-square and mean dispersal distances, respectively. The universal relation $\rho=\sqrt{2} R_{\mathrm{g}}$ directly connects the radius of gyration to the RMS dispersal distance. There is no such useful connection of $R_{*}$ to dispersion under random motion. Therefore, the radius of gyration is a measure of dispersal of an animal within a habitat cluster. Our calculations clearly demonstrate that the radius of gyration is the only metric, amongst the landscape metrics that we consider, that has an ecological interpretation and is independent of the cluster shape.

The term 'the radius of gyration' was introduced to the ecology literature by Keitt et al. (1997) and Riitters et al. (1995). However, their equations differ from each other, and from Eq. (2), meaning that they do not have an ecological interpretation. This has had an unfortunate consequence: the selection [following Keitt et al. (1997)] of what we call $R_{*}$ as the metric GYRATE in the widely used FRAGSTATS package (McGarigal et al. 2012). This is likely to be a source of ongoing 

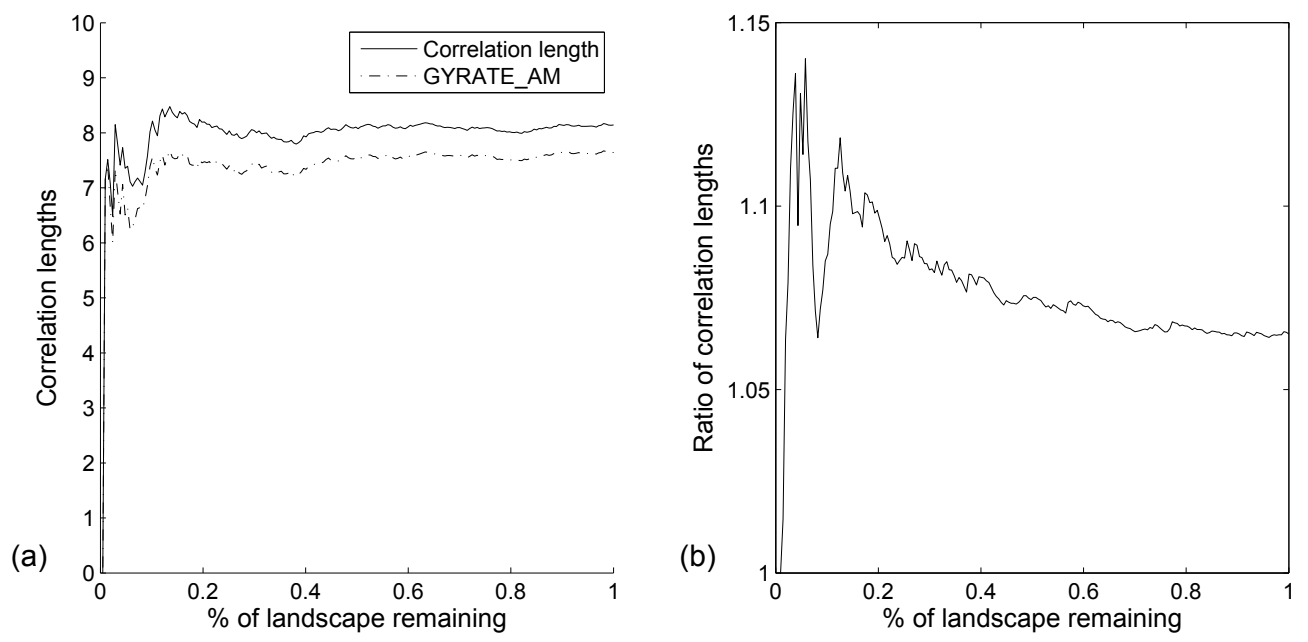

Fig. 3.- Comparison between the the two metrics $\xi$ (correlating length) and $\xi_{*}$ (GYRATE_AM) as patches are randomly removed from a fragmented habitat. The inital fragmented habitat was created by assigning the patches of a $20 \times 20$ piece of the square lattice independent probabilities 0.5 of being suitable habitat patches. Two habitat patches that are adjacent (including diagonal patches) are deemed to belong to the same cluster. (a) The correlation length $\xi$ (solid black line) and $\xi_{*}=$ GYRATE_AM (dashed black line) as patches are removed. (b) The ratio $\xi / \xi_{*}$ as patches are removed. The ratio is always greater than unity, apart from the trivial case (a single patch) when $\xi / \xi_{*}=1$.

confusion. Quantitative differences between $R_{\mathrm{g}}$ and $R_{*}=$ GYRATE can be substantial (Fig. 2). This also extends to FRAGSTATS version of the cluster-weighted average $\xi_{*}$. The difference between $\xi$ and $\xi_{*}$ is shown in Fig. 3. In this simulation, a random landscape is initially generated, then habitat patches are removed at random, and the two metrics are calculated and compared after each removal.

Consequences of ambiguous terminology are not merely quantitative, but also enter into interpretation. For example, Keitt et al. (1997) state that what we call $R_{*}$ represents both the average distance an individual is able to disperse before reaching a boundary and the average distance a randomly placed, randomly moving particle on a cluster will move before encountering the edge of a cluster. Neither of these interpretations are consistent with the definition of $R_{*}$ or $R_{g}$.

The question of the distance typically taken to move from a random location to a habitat boundary is quite a subtle one. Discretizing a habitat introduces a length scale that has no a priori meaning in terms of an organism's movements, and the actual path lengths depend strongly on the discretisation length scale. This is a simple manifestation of Mandelbrot's famous question "How long is the coast of Britain?" (Mandelbrot 1967, 1982). Similar issues arise with path lengths obtained by interpolating between positions observed at fixed time intervals and indeed for diffusion the path traced as an object moves from one location to other is a fractal curve of infinite length (Mandelbrot 1982). It may be more helpful to focus on statistics related to the time taken to move between points (the "first-passage time"), about which much is known for standard random processes (Redner 2001). This does not avoid model dependence, of course, because the distribution of first-passage times (and so mean values and so on) depends on the precise random process being considered. Some calculations of first-passage times for continuous regions are given in the Appendix. Characteristic length scales deduced from first-passage times exhibit functional dependence on geometric parameters such as aspect ratio that differ from the analogous dependence found for lengths computed directly from the geometry without any consideration of the nature of organism movement. 


\section{Conclusion}

Naive computation of measures based simply on the geometry of habitat is unlikely to produce landscape metrics of ecological significance. However, if one calculates enough of these metrics it may be possible to observe some empirical correlations between geometric attributes of habitats and field data on the presence of species (Vos et al. 2001). A number of authors have advocated the use of metrics that reveal underlying ecosystem processes, rather than just landscape patterns (Kupfer 2012; Moilanen \& Hanski 2001; White et al. 2014; Vos et al. 2001). In this note we have examined metrics that are calculated from the geometry of habitat patches to find which metrics have a useful ecological interpretation. The radius of gyration $R_{\mathrm{g}}$, defined by Eq. (2), as opposed to the definition $R_{*}=$ GYRATE in FRAGSTATS, may be the only purely geometry-based landscape metric with direct ecological significance.

\section{Acknowledgements}

We thank Dr Michael Bode, Dr Luke Kelly and Dr Michael McCarthy for helpful comments. We also thank the Australian Research Council Center of Excellence for Environmental Decisions, the National Environmental Research Project and The University of Melbourne for funding.

\section{Appendix: Length scales for plane regions}

We discuss several measures of length associated with finite plane regions. The first set of measures contains lengths arising from geometry alone and are the continuous analogues of the discrete patch-based definitions of Section 2. The second set of measures contains lengths arising from standard stochastic processes. In this continuum approach, habitat patches are represented by geometrical points.

\section{A.1 Geometrically defined lengths}

We consider a region $\Omega$ in the plane that is entirely contained within a circle of finite radius, and therefore having a finite area, denoted by $A$. The boundary of the region $\Omega$ is denoted by $\partial \Omega$. The area element for integration is denoted by $d S$ when integrating with respect to $\mathbf{r} \in \Omega$, and by $d S^{\prime}$ when integrating with respect to $\mathbf{r}^{\prime} \in \Omega$. The center of mass is

$$
\mathbf{r}_{\mathrm{CM}}=\frac{1}{A} \int_{\Omega} \mathbf{r} d S .
$$

The quantities $R_{\mathrm{g}}, R_{*}, B, B_{*}, \rho$ and $\rho_{*}$ defined below are positive and have dimensions of length:

(i) the standard version of the radius of gyration $R_{\mathrm{g}}$, given by ${ }^{\dagger}$

$$
R_{\mathrm{g}}^{2}=\frac{1}{A} \int_{\Omega}\left|\mathbf{r}-\mathbf{r}_{\mathrm{CM}}\right|^{2} d S
$$

(ii) a non-standard definition of a kind of radius of gyration,

$$
R_{*}=\frac{1}{A} \int_{\Omega}\left|\mathbf{r}-\mathbf{r}_{\mathrm{CM}}\right| d S
$$

(iii) the root-mean-square distance to boundary, $B$, given by

$$
B^{2}=\frac{1}{A} \int_{\Omega} \min _{\mathbf{r}^{\prime} \in \partial \Omega}\left|\mathbf{r}-\mathbf{r}^{\prime}\right|^{2} d S
$$

(iv) the mean distance to boundary,

$$
B_{*}=\frac{1}{A} \int_{\Omega} \min _{\mathbf{r}^{\prime} \in \partial \Omega}\left|\mathbf{r}-\mathbf{r}^{\prime}\right| d S
$$

(v) the root-mean-square distance between two points, $\rho$, given by

$$
\rho^{2}=\frac{1}{A^{2}} \int_{\Omega} \int_{\Omega}\left|\mathbf{r}-\mathbf{r}^{\prime}\right|^{2} d S d S^{\prime} ;
$$

(vi) the mean distance between two points,

$$
\rho_{*}=\frac{1}{A^{2}} \int_{\Omega} \int_{\Omega}\left|\mathbf{r}-\mathbf{r}^{\prime}\right| d S d S^{\prime} .
$$

Since for any random variable $X$ with finite variance, $\left\langle X^{2}\right\rangle \geqslant\langle X\rangle^{2}$, with strict inequality unless $X$ can take only one value, we have the strict inequalities

$$
R_{\mathrm{g}}>R_{*}, \quad B>B_{*}, \quad \rho>\rho_{*} .
$$

\footnotetext{
${ }^{\dagger}$ For standard shapes we can look up $R_{\mathrm{g}}$ from tables of the moment of inertia $I$ of a uniform lamina of mass $M$ about its center of mass, using $I=M R_{\mathrm{g}}^{2}$.
} 
It is easy to prove that

$$
\int_{\Omega} \int_{\Omega}\left|\mathbf{r}-\mathbf{r}^{\prime}\right|^{2} d S d S^{\prime}=2 A \int_{\Omega}\left|\mathbf{r}-\mathbf{r}_{\mathrm{CM}}\right|^{2} d S
$$

and it follows that $\rho^{2}=2 R_{\mathrm{g}}^{2}$ [cf. Eq. (8)], that is,

$$
\rho=\sqrt{2} R_{\mathrm{g}} .
$$

The pair of length scales $R_{\mathrm{g}}$ and $\rho$ is the only one of the 15 possible pairs of length scales defined in Eqs (10)-(15) for which there is an exact relation between the length scales that is independent of the domain shape. This can be seen from the exact calculations now performed for several simple domain shapes. Most of the integrals are easily evaluated, but in two cases the final exact evaluation of a double integral has been obtained with MATHEMATICA ${ }^{\ddagger}$ while in one case only a numerical evaluation with MAтHEMAтіCA was available.

\section{A.1.1 Special case-a circular disc of radius a}

With the center of mass at the disc center, then

$$
\begin{aligned}
& R_{\mathrm{g}}^{2}=\frac{1}{\pi a^{2}} \int_{0}^{a} 2 \pi r r^{2} d r=\frac{a^{2}}{2}, \\
& R_{*}=\frac{1}{\pi a^{2}} \int_{0}^{a} 2 \pi r r d r=\frac{2 a}{3}, \\
& B^{2}=\frac{1}{\pi a^{2}} \int_{0}^{a} 2 \pi r(a-r)^{2} d r=\frac{a^{2}}{6}, \\
& B_{*}=\frac{1}{\pi a^{2}} \int_{0}^{a} 2 \pi r(a-r) d r=\frac{a}{3} .
\end{aligned}
$$

Since $\rho=\sqrt{2} R_{\mathrm{g}}$, it remains only to compute $\rho_{*}$, and in this case the integration is not elementary. Where

$$
\left|\mathbf{r}-\mathbf{r}^{\prime}\right|=\sqrt{r^{2}-2 r r^{\prime} \cos \left(\theta-\theta^{\prime}\right)+\left(r^{\prime}\right)^{2}}
$$

we have

$$
\rho_{*}=\frac{1}{\left(\pi a^{2}\right)^{2}} \int_{0}^{a} \int_{0}^{a} \int_{0}^{2 \pi} \int_{0}^{2 \pi} r r^{\prime}\left|\mathbf{r}-\mathbf{r}^{\prime}\right| d \theta d \theta^{\prime} d r d r^{\prime} .
$$

Using the changes of variables $r=a x$ and $r^{\prime}=$ $a x^{\prime}$, setting $\theta=\theta^{\prime}+\phi$ in the inner integral and exploiting the periodicity of the integrand we find that

$$
\begin{array}{r}
\rho_{*}=\frac{2 a}{\pi} \int_{0}^{1} \int_{0}^{1} \int_{0}^{2 \pi} x x^{\prime} \sqrt{x^{2}-2 x x^{\prime} \cos (\phi)+\left(x^{\prime}\right)^{2}} \\
d \phi d x d x^{\prime} .
\end{array}
$$

After a little algebra we can evaluate the integral over $\phi$ in terms of the complete elliptic integral of the second kind $^{\S}$

$$
E(k)=\int_{0}^{\pi / 2} \sqrt{1-k^{2} \sin ^{2} \theta} d \theta,
$$

and we find that

$$
\begin{aligned}
\rho_{*} & =\frac{8 a}{\pi} \int_{0}^{1} \int_{0}^{1} x x^{\prime}\left(x+x^{\prime}\right) E\left(\sqrt{\frac{4 x x^{\prime}}{\left(x+x^{\prime}\right)^{2}}}\right) d x d x^{\prime} \\
& \approx 0.9054 a .
\end{aligned}
$$

Hence, listing the length scales in increasing order,

$$
\begin{aligned}
B_{*} & =\frac{a}{3} \approx 0.3333 a, & B & =\frac{a}{\sqrt{6}} \approx 0.4082 a, \\
R_{*} & =\frac{2 a}{3} \approx 0.6667 a, & R_{\mathrm{g}} & =\frac{a}{\sqrt{2}} \approx 0.7071 a, \\
\rho_{*} & \approx 0.9054 a, & \rho & =\sqrt{2} R_{\mathrm{g}}=a .
\end{aligned}
$$

\section{A1.1.2 Special case-a square of side $L$}

In Cartesian coordinates, the square corresponds to $0<x<L$ and $0<y<L$. With center of mass at $(L / 2, L / 2)$, we obtain

$$
\begin{aligned}
R_{\mathrm{g}}^{2} & =\frac{1}{L^{2}} \int_{0}^{L} \int_{0}^{L}\left[\left(x-\frac{L}{2}\right)^{2}+\left(y-\frac{L}{2}\right)^{2}\right] d x d y \\
& =\frac{L^{2}}{6} \\
R_{*} & =\frac{1}{L^{2}} \int_{0}^{L} \int_{0}^{L}\left[\left(x-\frac{L}{2}\right)^{2}+\left(y-\frac{L}{2}\right)^{2}\right]^{1 / 2} d x d y \\
& =\frac{L}{6}[\sqrt{2}+\operatorname{arcsinh}(1)] \approx 0.3826 L
\end{aligned}
$$

To compute $B^{2}$ and $B_{*}$, it suffices to take the average over the region $0<x<L / 2,0<y<x$, which has area $(1 / 2)(L / 2)^{2}=L^{2} / 8$, so that

$$
\begin{aligned}
& B^{2}=\frac{8}{L^{2}} \int_{0}^{L / 2} \int_{0}^{x} y^{2} d y d x=\frac{L^{2}}{24}, \\
& B_{*}=\frac{8}{L^{2}} \int_{0}^{L / 2} \int_{0}^{x} y d y d x=\frac{L}{6} .
\end{aligned}
$$

\footnotetext{
$\S$ There is an issue with notational conventions for the elliptic integrals that requires care in software use. We follow Whittaker \& Watson (1927) in defining $E(k)$, so that the MATHEMatica function which we use to evaluate the integral numerically is EllipticE $[m]=E(\sqrt{m})$.
} 
Again we note that the root-mean-square distance between points is $\rho=\sqrt{2} R_{\mathrm{g}}$. The computation of the average distance between points,

$$
\begin{array}{r}
\rho_{*}=\frac{1}{L^{4}} \int_{0}^{L} \int_{0}^{L} \int_{0}^{L} \int_{0}^{L} \sqrt{\left(x-x^{\prime}\right)^{2}+\left(y-y^{\prime}\right)^{2}} \\
d x d y d x^{\prime} d y^{\prime}
\end{array}
$$

is accomplished by setting $x=L X, y=L Y$, $x^{\prime}=L X^{\prime}$ and $y^{\prime}=L Y^{\prime}$, using symmetry to reduce the size of the integration region, followed by the additional transformations $u=X^{\prime}-X$, $v=Y^{\prime}-Y$, and appropriately chosen interchanges of orders of integration. We find that

$$
\rho_{*}=\frac{L}{15}[2+\sqrt{2}+5 \operatorname{arcsinh}(1)] \approx 0.5214 L .
$$

Hence, listing the length scales in increasing order,

$$
\begin{aligned}
B_{*} & =\frac{L}{6} \approx 0.1667 L, & B & =\frac{L}{\sqrt{24}} \approx 0.2041 L, \\
R_{*} & \approx 0.3826 L, & R_{\mathrm{g}} & =\frac{L}{\sqrt{6}} \approx 0.4082 L, \\
\rho_{*} & \approx 0.5214 L, & \rho & =\frac{L}{\sqrt{3}} \approx 0.5774 L .
\end{aligned}
$$

\section{A1.1.3 Aspect ratio effects}

From a standard text [Synge \& Griffith (1959), pp. 176, 292] we can extract the following formulae for the radius of gyration:

$$
\begin{array}{ll}
\text { rectangle of sides } a, b & R_{\mathrm{g}}^{2}=\frac{a^{2}+b^{2}}{12} ; \\
\text { ellipse of semiaxes } a \text { and } b & R_{\mathrm{g}}^{2}=\frac{a^{2}+b^{2}}{4} .
\end{array}
$$

The integrations needed to verify these results are straightforward. It is also easy to see (using symmetry) that for a rectangle of sides $a$ and $b$, with $a \geqslant b$, that the $k$ th power of the shortest distance to the boundary has the integral representation

$$
\frac{4}{a b}\left\{2 \int_{0}^{b / 2} \int_{0}^{y} x^{k} d x d y+\int_{0}^{b / 2} \int_{b / 2}^{a / 2} y^{k} d x d y\right\}
$$

from which it follows that

$$
B_{*}=\frac{(3 a-b) b}{12 a}, \quad B=\left[\frac{2 a b^{2}-b^{3}}{24 a}\right]^{1 / 2} .
$$

Hence for the rectangle the aspect ratio $b / a$ enters into $R_{\mathrm{g}}, B_{*}$ and $B$ in a different way: even for simple shapes one or two metrics alone provide very limited information.

\section{A1.2 Lengths from stochastic processes}

We may describe the dispersal distance of an organism as the straight-line distance between its original and current positions. If we model the dispersal by a diffusion process in $\Omega$ with reflecting (that is, zero flux) boundary conditions, then after a significant time has elapsed the organism's position is uniformly distributed over the region. If we also average over the original position of the organism we find that

mean dispersal distance

$=$ mean distance between points in $\Omega$

$=\rho_{*}$,

mean-square dispersal distance

$=$ mean-square distance between points in $\Omega$

$=\rho^{2}$,

where the lengths $\rho_{*}$ and $\rho$ were defined in Eqs (15) and (14) respectively. We now find that the radius of gyration $R_{\mathrm{g}}$ is connected, in a way independent of region shape or size, to two other lengths, one defined solely by geometry, and one having significance for dispersal:

radius of gyration

$$
\begin{aligned}
& =\sqrt{2} \times\left\{\begin{array}{l}
\text { root-mean-square distance } \\
\text { between points }
\end{array}\right\} \\
& =\sqrt{2} \times \text { root-mean-square dispersal distance. }
\end{aligned}
$$

The non-standard definition of a kind of radius of gyration [denoted here by $R_{*}$ : see Eq. (11)] does not enter into such shape-independent relations.

An alternative measure of distance associated with a domain is the length of the path traced out by an organism as it moves from its original location to its first encounter with the boundary. The computation of this distance is very sensitive to the precise stochastic model used, and in the case of diffusion, the distance is mathematically infinite, due to the extreme irregularity of individual realizations of Brownian motion. A better measure is the time taken to reach the boundary for the first time, often called the first-passage time.

For a point particle that undergoes a twodimensional Pólya-type random walk on the square lattice (Hughes 1995) but with lattice spacing $\Delta$ and time between steps $\tau$ we have an evolution equation for the occupancy probabilities 
$P(x, y, t)$ and for the mean first-passage time to the boundary, $T(x, y)$ :

$$
\begin{aligned}
& P(x, y, t+\tau) \\
& =\frac{1}{4}[P(x+\Delta, y, t)+P(x-\Delta, y, t) \\
& \quad+P(x, y+\Delta, t)+P(x, y-\Delta, t)], \\
& T(x, y) \\
& =\tau+\frac{1}{4}[T(x+\Delta, y)+T(x-\Delta, y) \\
& \quad+T(x, y+\Delta)+T(x, y-\Delta)] .
\end{aligned}
$$

Proceeding to the continuum limit, where

$$
D=\lim _{\Delta \rightarrow 0, \tau \rightarrow 0} \Delta^{2} /(4 \tau)
$$

(and scaling $P$ with $\Delta^{2}$ appropriately) we find that

$$
\frac{\partial P}{\partial t}=D \nabla^{2} P, \quad D \nabla^{2} T+1=0 .
$$

We can compute first-passage time statistics by solving the diffusion equation with the boundary condition $P=0$ for a delta function initial condition, and then doing appropriate integrals, or we can just solve the Poisson equation for $T$ with $T=0$ on the boundary to get the mean first passage time directly (Redner (2001), p. 31).

\section{A1.2.1 Mean first-passage time for an ellipse}

For an ellipse with semiaxes $a$ and $b$, represented by $x^{2} / a^{2}+y^{2} / b^{2} \leqslant 1$,

$$
T(x, y)=K\left(1-\frac{x^{2}}{a^{2}}-\frac{y^{2}}{b^{2}}\right)
$$

where

$$
K=\frac{a^{2} b^{2}}{2 D\left(a^{2}+b^{2}\right)} .
$$

Averaging over the starting location gives the mean first-passage time from a random starting location as

$$
\bar{T}=\frac{1}{\pi a b} \iint_{\text {ellipse }} K\left(1-\frac{x^{2}}{a^{2}}-\frac{y^{2}}{b^{2}}\right) d x d y .
$$

Setting $x=a X$ and $y=b Y$ and then changing to polar coordinates, we find that

$$
\bar{T}=\frac{a^{2} b^{2}}{4 D\left(a^{2}+b^{2}\right)}=\frac{\ell^{2}}{D}
$$

where

$$
\ell=\left[\frac{a^{2} b^{2}}{4\left(a^{2}+b^{2}\right)}\right]^{1 / 2} .
$$

This introduces a new length scale not related to other length scales except for a circle when $a=b$.

\section{A1.2.2 Mean first-passage time for a rectangle}

For the rectangle of sides $a$ and $b$, represented in Cartesian coordinates by $0 \leqslant x \leqslant a, 0 \leqslant y \leqslant b$, the standard trial solution based on the eigenfunctions of the Laplace operator with homogeneous Dirichlet boundary conditions and the use of standard results from the theory of Fourier series (Pinchover \& Rubinstein 2005) leads to the solution

$$
\begin{aligned}
T(x, y)= & \frac{16}{\pi^{4} D} \sum_{m=0}^{\infty} \sum_{n=0}^{\infty} \frac{1}{(2 m+1)(2 n+1)} \\
& \times \frac{\sin [(2 m+1) \pi x / a] \sin [(2 n+1) \pi y / b]}{\left[(2 m+1)^{2} / a^{2}+(2 n+1)^{2} / b^{2}\right]} .
\end{aligned}
$$

Averaging over the starting location, we find that

$$
\begin{aligned}
\bar{T}= & \frac{1}{a b} \int_{0}^{b} \int_{0}^{a} T(x, y) d x d y \\
= & \frac{64}{\pi^{6} D} \sum_{m=0}^{\infty} \sum_{n=0}^{\infty} \frac{1}{(2 m+1)^{2}(2 n+1)^{2}} \\
& \times \frac{1}{\left[(2 m+1)^{2} / a^{2}+(2 n+1)^{2} / b^{2}\right]} \\
= & \frac{\ell^{2}}{D} .
\end{aligned}
$$

Again $\ell$ is not related to any length scale we have discussed, except for the square when $a=b$.

\section{A1.2.3 Effect of aspect ratio on mean first-passage} times

It is instructive to write the mean first-passage time for the ellipse and the rectangle in the form

$$
\bar{T}=\frac{A}{D} f(b / a),
$$

where $A$ is the area ( $\pi a b$ and $a b$ for the ellipse and rectangle, respectively). The dimensionless function $f(b / a)$ gives the dependence of the domain on the aspect ratio $b / a$. There is no loss of generality in assuming that $a \geqslant b$, so that $b / a \leqslant 1$. The exact formulae for $f(b / a)$ are

$$
f(b / a)=\frac{1}{4 \pi(a / b+b / a)}
$$




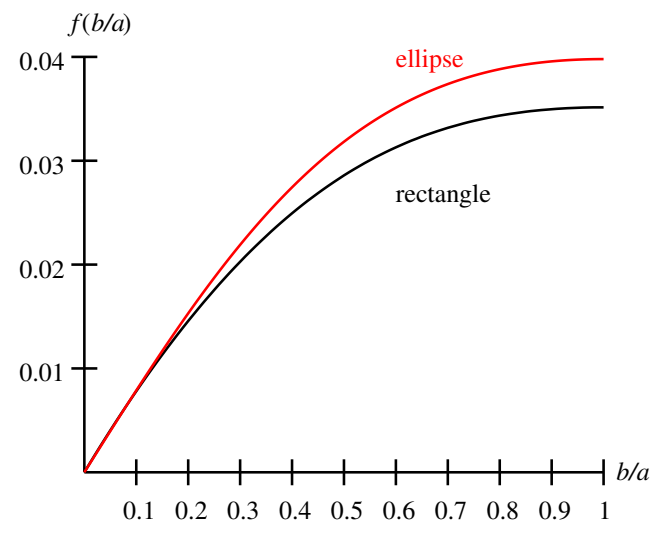

Fig. 4.- The function $f(b / a)$ captures the aspect ratio dependence of the mean first-passage time from a random interior point to the boundary for an ellipse with semiaxes $a$ and $b$ (upper red curve) and for a rectangle with sides $a$ and $b$ (the lower black curve) where $a \geqslant b$.

for the ellipse, and

$$
\begin{aligned}
f(b / a)= & \frac{64}{\pi^{6}} \sum_{m=0}^{\infty} \sum_{n=0}^{\infty} \frac{1}{(2 m+1)^{2}(2 n+1)^{2}} \\
& \times \frac{1}{\left[(2 m+1)^{2} b / a+(2 n+1)^{2} a / b\right]}
\end{aligned}
$$

for the rectangle. These functional forms are illustrated in Fig. 4.

\section{REFERENCES}

Brissonette, J.A. 1997. Wildlife and landscape ecology - effects of pattern and scale. Springer.

Creswick, R.J., H.A. Farach, C.P. Poole, 1992. Introduction to renormalization group methods in physics. Wiley, New York.

Duraiappah, A.K., S. Naeem, and T. Agardy. 2005. Millennium ecosystem assessment, 2005. Ecosystems and human well-being: biodiversity synthesis, millennium ecosystem assessment. World Resources Institute, Washington, DC.

Ewers, R.M., and R.K. Didham. 2007. The effect of fragment shape and species sensitivity to habitat edges on animal population size. Conservation Biology 21:926-936.
Feder, J., 1988. Fractals, Physics of Solids and Liquids. Springer US.

Hargis, C.D., J.A. Bissonette, and J.L. David. 1998. The behavior of landscape metrics commonly used in the study of habitat fragmentation. Landscape Ecology 13:167-186.

Hughes, B.D., 1995. Random walks and random environments: Volume 1: random walks. Oxford University Press, USA.

Hughes, B.D., 1996. Random walks and random environments: Volume 2: random environments. Oxford University Press, USA.

Keitt, T.H., D.L. Urban, and B.T. Milne. 1997. Detecting critical scales in fragmented landscapes. Conservation Ecology 11955449 1, 4-4.

Kupfer, J.A., 2012. Landscape ecology and biogeography Rethinking landscape metrics in a post-FRAGSTATS landscape. Progess in Physical Geography. 36, 400-420.

Li, H., and J. Wu. 2004. Use and misuse of landscape indices. Landscape Ecology 19: 389-399.

Mandelbrot, B.B. 1967, How long is the coast of Britain? Statistical self-similarity and fractional dimension, Science 155: 636-638.

Mandelbrot, B.B. 1982. The fractal geometry of nature. Freeman, San Francisco.

McGarigal, K., S.A. Cushman, and E. Ene. 2012. FRAGSTATS v4: Spatial pattern analysis program for categorical and continuous maps.

Moilanen, A., and I. Hanski. 2001. On the use of connectivity measures in spatial ecology. Oikos 95:147-151.

Pinchover, Y., and J. Rubinstein. 2005. An introduction to partial differential equations. Cambridge University Press.

Redner, S. 2001, A guide to first-passage processes. Cambridge University Press.

Riitters, K.H., R.V. ONeill, C.T. Hunsaker, J.D. Wickham, D.H. Yankee, S.P. Timmins, K.B. Jones, and B.L. Jackson. 1995. A factor analysis of landscape pattern and structure metrics. Landscape Ecology 10: 23-39. 
Stauffer, D., and A. Aharony,. 1994. Introduction to percolation theory, revised second edition. Taylor and Francis, London.

Synge, J.L., Griffith, B.A., 1959. Principles of Mechanics, 3rd ed. McGraw-Hill, New York.

Vos, C.C., J. Verboom, P.F.M. Opdam, and C.J.F. Ter Braak. 2001. Toward ecologically scaled landscape indices. American Naturalist 157: $24-41$.

White, J.W., J. Schroeger, P.T. Drake, and C.A. Edwards. 2014. The value of larval connectivity information in the static optimization of marine reserve design. Conservation Letters. doi:10.1111/conl.12097.

Whittaker. E.T., and G.N. Watson. 1927. A course of modern analysis, 4th edition, Cambridge University Press.

Wu, J., 2013. Landscape ecology. In: Leemans, R. (Ed.), Ecological Systems. Springer, New York, pp. 179-200.

This 2-column preprint was prepared with the AAS IATEX macros v 5.2 . 


\section{University Library}

\section{- M M I N E R VA A gateway to Melbourne's research publications}

Minerva Access is the Institutional Repository of The University of Melbourne

Author/s:

Baker, CM;Hughes, BD;Landman, KA

Title:

Length-based connectivity metrics and their ecological interpretation

Date:

2015-11-01

Citation:

Baker, C. M., Hughes, B. D. \& Landman, K. A. (2015). Length-based connectivity metrics and their ecological interpretation. ECOLOGICAL INDICATORS, 58, pp.192-198. https:// doi.org/10.1016/j.ecolind.2015.05.046.

Persistent Link:

http://hdl.handle.net/11343/58817 